\title{
Fuzzy Control for Dual-stator Toroidal Motor with Hybrid Excitation
}

\author{
LIU Xin, WANG Yang Yang
}

Tianjin key laboratory of modern electromechanical equipment technology

Tianjin polytechnic university, Tianjin,China

liuxin@tjpu.edu.cn

Keywords: Fuzzy Control; Dual-stator; Toroidal Motor; Hybrid Excitation.

\begin{abstract}
Dual-stator toroidal motor with hybrid excitation is a new-style motor, it integrates power and drive function. The mathematic model of the motor system was derived based on the operating principle of the motor. In order to improve the response characteristic of toroidal motor, a fuzzy self-tuning PID control system was presented and a fuzzy controller was designed based on fuzzy control rules. The response of fuzzy control system with disturbance was simulated. The comparison results justify the better anti-interference ability of the toroidal motor with fuzzy control system. The research is useful for further controller design and practical application of the novel motor.
\end{abstract}

\section{Introduction}

Dual-stator toroidal motor with hybrid excitation integrates power and drive, it can transmit large torque in a small size. The integration of mechanical elements and electromagnetic ones makes the control easier, at the same time, the motor also assemble with the decelerator. So the dual-stator toroidal motor is suitable for the fields such as robots, aviation and space flight[1,2]. The servo system can be substituted by toroidal motor to simplify the structure of electromechanical system[3]. As more and more electrical and control techniques are utilized in power engineering field, new-style motors become advancing edge of electromechanical science. Thus the dual-stator toroidal motor with hybrid excitation has more expansive application prospect.

Figure 1 shows the schematic diagram of the dual-stator toroidal motor. The novel motor consists of four basic elements: (1) the worm inner stator; (2) radially positioned planets; (3) outer stator; and (4) rotor upon which the planets are mounted. Among them, the inner stator is fixed and the armature coils are mounted in helical slots of its surface. The planets adopt permanent magnets teeth for excitation, the $\mathrm{N}$ and $\mathrm{S}$ pole permanent magnets mounted alternately on each planet. Outer stator has several helical magnetic steel or alternating NS pole permanent magnets embedded in the bracket. When a specific relationship is satisfied between the planet pitch, lead angle of outer stator and the pole-pairs number of inner stator, the $\mathrm{N}$ pole of one element will corresponding to $\mathrm{S}$ pole of the other elements.

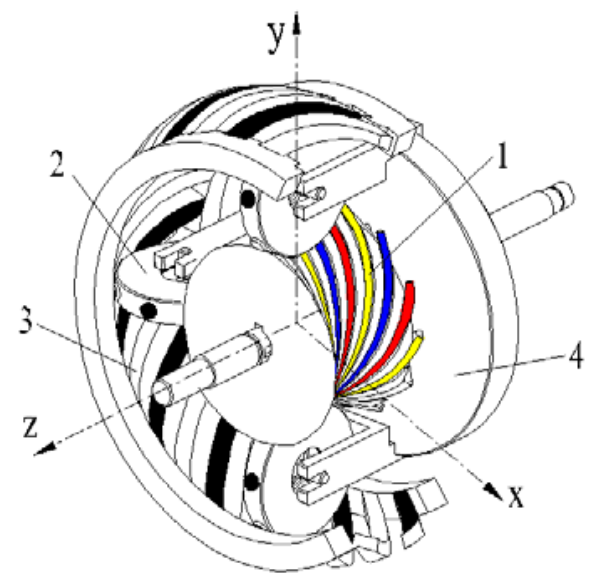

Fig.1 Schematic diagram of toroidal motor 
When the alternating current is connected to the coils of the armature worm, a toroidal circular electromagnetic field is formed. The magnetic forces between stators and rotor drive the planet teeth run along with helical trajectory, that is, the planets rotate about their own axes, meanwhile the rotor rotates about its own axis. So the output with a large torque at low speed is generated. In this paper, according to the electromechanical coupled model of the toroidal motor, the mathematic model of the speed control for the motor is derived. In order to enhance the robustness of the drive system, the fuzzy control rules are formulated based on the control theory and experience. A fuzzy self-tuning PID control system is designed based on the fuzzy rules. The response of speed control system is simulated. The results are useful for designing and manufacturing the controller for the novel motor.

\section{Mathematic Model of the Motor}

The Electromechanical coupled toroidal motor system consists of a worm stator, outer stator and a rotor with planets. They couple with each other by magnetic field caused by currents and magnetic teeth. The voltage differential equation of the worm coil is

$$
v_{s}=R_{s} i_{s}+\frac{d \psi_{s}}{d t}
$$

Where $R_{\mathrm{s}}$ and $i_{\mathrm{s}}$ are resistance and current of worm coils respectively. The magnetic linkage $\psi_{s}=1.5 L_{s 0} i_{s}+\psi_{f} \sin \theta_{e}, L_{\mathrm{s} 0}$ is the average value of fundamental wave self-inductance of the worm coils, $\psi_{f}$ is mutual-inductance magnetic linkage between planet teeth and the worm coil, $\theta_{e}$ which is the autorotation electric angle of planet has the transmission relationship $\theta_{\mathrm{e}}=Z_{0} \theta / 2, Z_{0}$ is tooth number of outer stator, $\theta$ is output rotating angle of the toroidal motor.

Substituting magnetic linkage into the voltage differential equation, yields

$$
v_{s}=L_{s} \&+R_{s} i_{s}+Z_{0} \psi_{s} \omega \cos \left(Z_{0} \theta / 2\right) / 2
$$

The motion equation of the toroidal driven system can be given as $T=K_{0} B_{r}+K_{1} i_{s}=J \&_{+} b{ }_{4}$, Where $B_{r}$ is the remnant magnetism of permanent magnet beam of the outer stator, $K_{0}$ and $K_{1}$ are the torque constant related to the structure of the outer and inner stator respectively. $J$ and $b$ are the equivalent rotary inertia and damping coefficient of the rotor.Combine with Eq.(2), following equation can be obtained after Laplace transform

$$
\left\{\begin{array}{l}
V_{s}-C \omega=\left(L_{s} s+R_{s}\right) I_{s} \\
K_{0} B_{r}+K_{1} I_{s}=(J S+b) \omega
\end{array}\right.
$$

Where $\mathrm{C}$ is the back electromotive force coefficient. In order to improve the output performance of toroidal motor, $v_{\mathrm{s}}$ is usually chosen as controllable input. the mathematic model is given as

$$
G_{s}=\frac{K_{1}}{J L_{s} s^{2}+\left(L_{s} b+J R_{s}\right) s+\left(R_{s} b+C K_{1}\right)}
$$

Considering the impact of some uncertain factors of toroidal motor, the parameters of the model may change. The controller designed according to the initial drive system can not meet the expected control effect, so the self-tuning control system has a certain practical significance.

\section{Fuzzy Control for Toroidal Motor}

Fuzzy control strategy obtains control signal by means of input fuzziness, process based on the fuzzy rule, and output anti-fuzzy at last. In the fuzzy self-tuning PID control system[4,5], the deviation and the first derivative of the deviation of the closed-loop system are the inputs of fuzzy controller, $K_{\mathrm{p}}, K_{\mathrm{i}}$ and $K_{\mathrm{d}}$ are the outputs of fuzzy controller. When the inputs change, the output parameters of fuzzy controller would adjust automatically according to the fuzzy rules which based on the control theory and experience. Figure 3 shows the fuzzy self-tuning PID control system. 


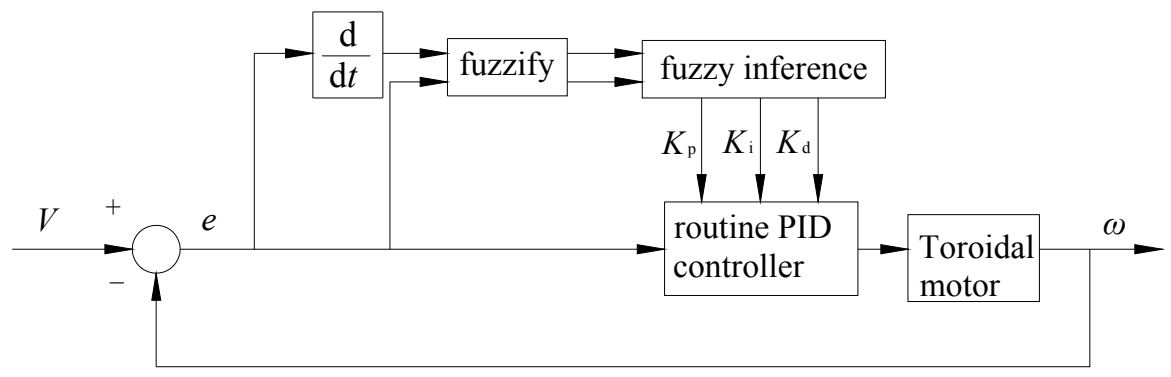

Fig.3. Fuzzy self-tuning PID control system

For the dual-stator toroidal motor, two-dimensional fuzzy controller is selected. The determine of the range of the input and output variables is extremely important. Too large or too small range for input variables will not only affect the stability of the closed-loop control system, but also increase the burden on the fuzzy controller. Output variables will affect the complexity of the hardware circuit. The ranges are divided into different intervals as fuzzy subsets according to the membership, the input and output fuzzy subsets of fuzzy control sysytem for toroidal motor are obtained respectively. Based on the experience and control strategy, the changes of output variables along with input variables are developed as fuzzy rule for toroidal motor.

In order to simulate the fuzzy control for the toroidal motor, the FIS file which embed fuzzy rules need to be completed for fuzzy controller, so the controller contains fuzzy PID control algorithm. The membership function between fuzzy subsets in file is shown in Figure 4. After editing fuzzy rules statement, the file needs to be imported into workspace, and then will be embedded directly in fuzzy logic controller, so the import of fuzzy rules for fuzzy control is realized.

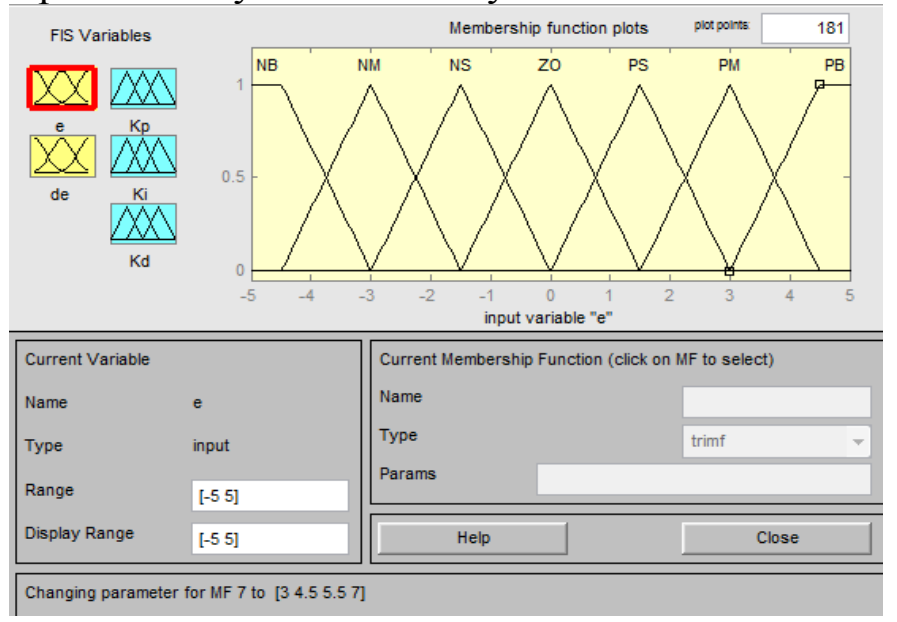

Fig.4. Membership function

Figure 5 shows the fuzzy control simulation model for toroidal motor. The main parameters of simulation for the controlled motor are given as $L_{\mathrm{s}}=0.1 \mathrm{H}, R_{\mathrm{s}}=20 \Omega, b=0.06 \mathrm{~kg} \cdot \mathrm{m}^{2} / \mathrm{s}, J=0.02 \mathrm{~kg} \cdot \mathrm{m}^{2}$, $Z_{0}=20$. In the internal structure of fuzzy controller, the initial parameters of the fuzzy PID module are selected from conventional PID parameters. For the purpose of contrast analysis, the fuzzy control system is combined with routine control in simulation.

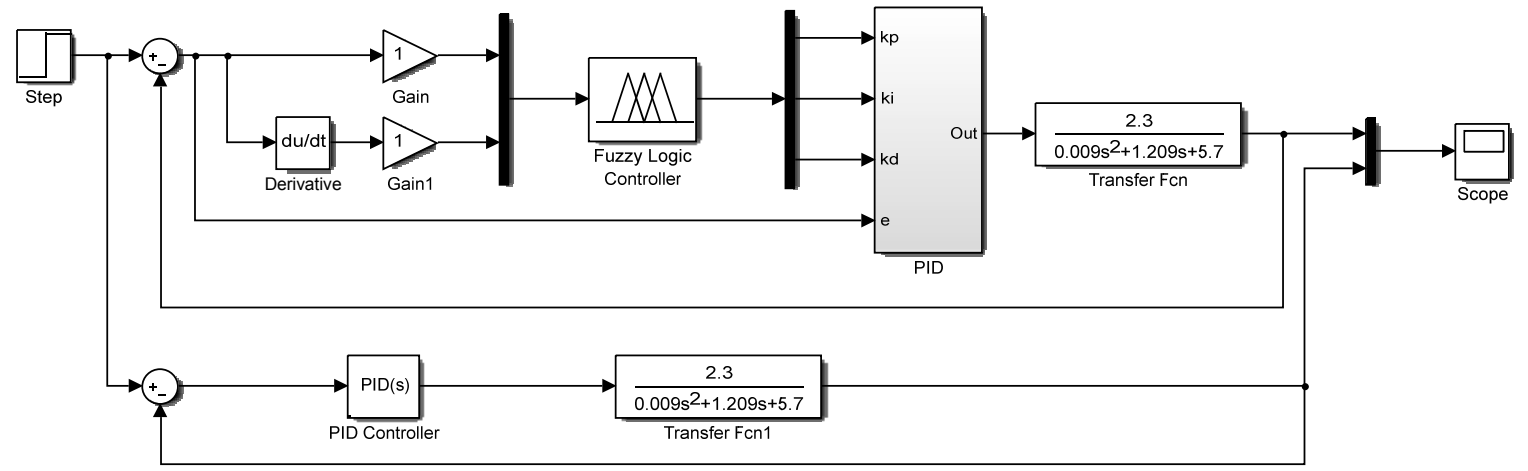

Fig.5. Fuzzy control system simulation 


\section{Results and Discussion}

Based on the simulation model above for toroidal motor, the simulation is carried out after the control parameters are set. The response to unit step signal of the closed-loop fuzzy control system can be easily obtained with simulation software. When the disturbance signal is added in the steady state of the system, the anti-disturbance capacity of the fuzzy control system can be analyzed. Figure 5 shows the simulation results for comparison.

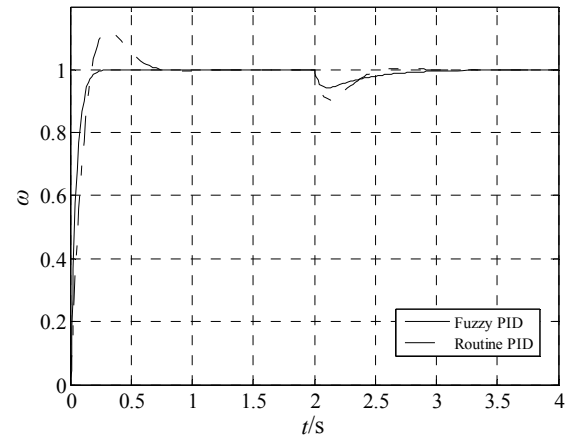

Fig.5. The simulation results for toroidal motor

In Fig.5, solid line represents the response of fuzzy self-tuning PID control for toroidal motor, and the dotted line represents the response of routine PID control. It is kown that the adjustment time $0.7 \mathrm{~s}$ and maximum overshoot $12 \%$ for routine control, while the adjustment time $0.2 \mathrm{~s}$ without overshoot for fuzzy control. Under the same disturbance, the system with fuzzy control suffers shock about $2.5 \%$ versus $11 \%$ of conventional PID control. From the comparison results, it is obvious that fuzzy PID control system has better signal tracking and stronger anti-interference ability.

\section{Summary}

In view of the electromechanical coupled model of dual-stator toroidal motor system, the mathematic model of the drive system was derived. The fuzzy self-tuning control system was presented, and a fuzzy controller was designed based on fuzzy control rules. Automatic adjustment of the controller parameters was realized.The simulation results show that the robustness ability of toroidal motor system was improved. These research results are meaningful for practical application of the motor.

\section{Acknowledgements}

This work was financially supported by the National Natural Science Foundation of China (No.51207107), and Innovation and Entrepreneurship Training Program of Tianjin polytechnic university (201410058125).

\section{References}

[1] Xu L Z, Hao X H, Dynamic model of electromechanical integrated toroidal drive. Applied Electromagnetics and Mechanics. 22(2005) 199-211.

[2] L. Xu, X. Liu, Optimal Control of the Electromechanical Integrated Toroidal Drive. Mechanical and Machine Theory. 44(2009) 1689-1700.

[3] M. Huichen. Parameter Analysis on Permanent-Magnet AC Servomotors, J. Micro machine. 38(2005) 3-6.

[4] B. Hamane, M. Benghanemm, etal. Control for variable speed wind turbine driving a doubly fed induction generator using Fuzzy-PI control. Energy Procedia, 18(2012) 476-485.

[5] S. Chekkal, N. Aouzellag Lahaçani, etal. Fuzzy logic control strategy of wind generator based on the dual-stator induction generator. Electrical Power and Energy Systems, 59(2014) 166-175. 